Beatriz Alves de Castro Barros

\title{
ASSIMETRIA INTERLATERAL DA ATENÇÃO VISUOESPACIAL VOLUNTÁRIA
}

São Paulo 2007 


\section{RESUMO}

\section{Castro-Barros, B. A. Assimetria interlateral da atenção visuoespacial voluntária.}

2007. 102 f. Tese (Doutorado em Ciências) - Instituto de Ciências Biomédicas, Universidade de São Paulo, São Paulo, 2007.

Há evidências que os mecanismos responsáveis pela atenção visuoespacial estão assimetricamente representados nos dois hemisférios cerebrais. Nesse estudo, a existência de uma diferença interlateral da orientação da atenção voluntária foi investigada. Uma tarefa de escolha simples foi utilizada nos Experimentos $1 \mathrm{~A}, 2 \mathrm{~A}$ e $3 \mathrm{~A}$ e uma tarefa de escolha vai/não-vai foi utilizada nos Experimentos $1 \mathrm{~B}, 2 \mathrm{~B}$ e $3 \mathrm{~B}$. Estímulos pistas centrais unilaterais (indicando lado esquerdo ou direito do espaço) e estímulos pistas centrais bilaterais (indicando ambos os lados do espaço) foram utilizados para direcionar a atenção. Tempos de reação a um alvo quando as pistas eram válidas, inválidas e bilaterais foram avaliados. As assincronias entre o início dos estímulos (AIE) foram de 100, 200, 300, 400 ou $500 \mathrm{~ms}$. Um importante efeito atencional (diferença entre o tempo de reação ao alvo quando a pista era inválida e o tempo de reação ao alvo quando a pista era válida) foi observado já aos $100 \mathrm{~ms}$ de AIE em todos os experimentos. No experimento $2 \mathrm{~B}$ este efeito foi maior quando a pista indicava o lado direito do que quando indicava o lado esquerdo, para a AIE de $500 \mathrm{~ms}$. Nos experimentos 3A e 3B, em que um distraidor ocorria simultaneamente com o alvo, o efeito atencional foi maior quando a pista indicava o lado direito do que quando indicava o lado esquerdo do espaço, para todas as AIE. Ainda nestes mesmos experimentos, quando a pista ocorria bilateralmente, o tempo de reação foi menor no lado direito do que no lado esquerdo, para todas as AIE. Os resultados indicam que a atenção voluntária tende a favorecer o lado direito do espaço particularmente quando a discriminação da forma do alvo é requerida.

Palavras-chave: Psicologia. Atenção Visual. Assimetria Interlateral. Tempo de Reação. 


\begin{abstract}
Castro-Barros, B.A. Interlateral asymmetry of visuospatial voluntary attention. 2007. 102 f. Doctorate Thesis - Instituto de Ciências Biomédicas, Universidade de São Paulo, São Paulo, 2007.

There is evidence that the mechanisms responsible for visuospatial attention are asymmetrically represented in the two cerebral hemispheres. In this study the existence of an interlateral difference of voluntary attention was investigated. A simple choice reaction time task was used in Experiments $1 \mathrm{~A}, 2 \mathrm{~A}$ and $3 \mathrm{~A}$ and a go/no-go choice reaction time task, in Experiments 1B, $2 \mathrm{~B}$ and $3 \mathrm{~B}$. Unilateral central cues (indicating left side or right side) and bilateral central cue (indicating both sides of space) were used to control attention. Reaction time to a visual target stimulus when the cue was valid, invalid or bilateral was evaluated. Stimulus onset asynchronies (SOA) were 100, 200, 300, 400 and 500 ms. An important attentional effect (difference between reaction time when the cue was invalid minus when the cue was valid) was observed as early as $100 \mathrm{~ms}$ in all experiments. In Experiments $2 \mathrm{~B}$ this effect was larger when the cue indicated the right side than when it indicated the left hemifield for SOA 500 ms. In Experiments 3A and 3B, when the distractor occurred simultaneously with the target, the attentional effect was larger when the cue indicated the right side than when it indicated the left side. In these same experiments, when the cue occurred bilaterally, reaction time was faster on the right hemifield, in all SOA. These results suggest that voluntary attention tends to favor the right side of space particularly when discrimination of the shape of the target stimulus is required.
\end{abstract}

Key Words: Psycology. Visual atention. Interlateral Asymmetry. Reaction Time. 


\section{INTRODUÇÃO}

A atenção é a atividade gerada por certas estruturas neurais que facilita 0 processamento de uma ou algumas informações sensoriais e inibe o processamento das demais informações sensoriais. Acredita-se que quando um estímulo é atendido sua representação neural seja facilitada e aquelas dos demais estímulos sejam inibidas. Desta forma o estimulo atendido pode ser identificado rapidamente e com acurácia. A habilidade de selecionar eventos sensoriais no espaço é um dos fatores cruciais para o comportamento adaptativo (GAZZANIGA, IVRY e MANGUN, 2002).

A orientação da atenção pode ocorrer de modo automático ou de modo voluntário. A orientação automática ocorre na vigência de estímulos salientes sensorialmente ou relevantes para o indivíduo, como estímulos súbitos, intensos ou em movimento (a sirene de uma ambulância, por exemplo), estímulos que contrastam com o fundo (uma cruz vermelha em uma parede braanca, por exemplo), ou estímulos significativos em um dado contexto (a mensão do próprio nome por outra pessoa, por exemplo). Tais características induzem um processamento sensorial cognitivo mais intenso, resultando no acionamento automático dos mecanismos atencionais. A orientação voluntária da atenção ocorre na vigência de motivações fisiológicas (sinais das vísceras), sociais (sugestões verbais) ou culturais (conhecimentos) (JONIDES, 1981; LAMBERT e HOCKEY, 1991; WARNER et al., 1990; THEEUWES, 1991).

A atenção automática e a voluntária podem ser avaliadas de modo quantitativo por meio dos testes de visão periférica, também chamado Procedimento de Posner (POSNER,1978; POSNER e RAICHLE, 1994). Nestes testes é possível aferir as alterações na latência da resposta e na acurácia que a atenção provoca. Em um experimento típico, uma tentativa inicia-se como o aparecimento de um ponto no centro da tela, chamado de ponto de fixação e de duas posições demarcadas na periferia. O voluntário deve manter os olhos fixos neste ponto durante toda a tentativa. Após um tempo variável é apresentado um estímulo (chamado estímulo precedente ou pista) que deve mobilizar a atenção para a periferia do campo visual. Algumas dezenas ou centenas de missegundos depois é apresentado, no local atendido ou em um local afastado deste, um outro estímulo (chamado de alvo) que deve ser apenas detectado ou também identificado. A resposta a este estímulo consiste em geral na pressão de uma tecla com o dedo indicador. $\mathrm{Na}$ atenção automática este primeiro estímulo é periférico (por exemplo, a 
mudança do brilho de uma das posições demarcadas) e não informativo espacialmente, isto é, ele indica corretamente o local de aparecimento do alvo em apenas metade das vezes. É observado, em intervalos curtos entre os estímulos (50 a $150 \mathrm{~ms}$ ), um menor tempo de reação quando o alvo ocorre na mesma posição (condição mesma) que a do estímulo precedente, e um maior tempo de reação quando o alvo ocorre na posição oposta àquela do estímulo precedente (condição oposta). Há uma facilitação das respostas do local previamente estimulado (condição mesma) e, uma inibição das respostas no local oposto ao estimulado (condição oposta). A soma do ganho de tempo na condição mesma mais a perda de tempo na condição oposta corresponde ao efeito atencional automático. Para intervalos maiores, de 200 a 1500 ms, foi observado que se desenvolvia uma inibição para o local previamente estimulado, fenômeno conhecido como inibição de retorno.

Na atenção voluntária este primeiro estímulo é central (por exemplo, uma seta apresentada no centro do campo visual) e informativo, isto é, na maioria das vezes ele indica corretamente o local de aparecimento do alvo (pista válida, por exemplo, $70 \%$ das vezes) e na minoria das vezes outro local (pista inválida, por exemplo, 30 \% das vezes). É observado um menor tempo de reação quando a pista é válida, e um maior tempo de reação quando a pista é inválida. Há uma facilitação das respostas no local indicado (pista válida) e uma inibição das respostas no local oposto àquele indicado (pista inválida). A soma do ganho de tempo quando a pista é válida mais a perda de tempo quando a pista é inválida corresponde ao efeito atencional voluntário.

O curso temporal da atenção automática e voluntária pode ser estudado variando-se a assincronia entre o início dos estímulos precedente e alvo (AIE) com ou sem variação correspondente da duração do estímulo precedente (POSNER et al., 1984; CHEAL, 1993; STEINMAM e STEINMAM, 1998). Diferentes cursos temporais da atenção têm sido descritos na literatura. Estudos que avaliaram a atenção automática mostraram que a facilitação no local previamente estimulado surge precocemente e atinge seu ápice após 50 de AIE (STEINMAM e STEINMAM, 1998; CASTRO-BARROS, 2004, NAKAYAMA e MACKEBEN, 1989; WARNER, JUOLA e KOSHINO, 1990; LYON, 1990; CHEAL, 1993; CHEAL, LYON e HUBBARD, 1990; CHEAL e LYON, 1991) e em seguida tente a permanecer constante (CASTRO-BARROS, 2004) ou diminuir (STEINMAM e STEINMAM, 1998; 
NAKAYAMA e MACKEBEN, 1989; CHEAL e LYON, 1991). A inibição no local oposto ao estimulado surge precocemente e permanece constante (CASTROBARROS, 2004; STEINMAN e STEIMNAN, 1998) ou aumenta progressivamente (WARNER, JUOLA e KOSHINO, 1990). Por outro lado, estudos que avaliaram a atenção voluntária mostraram que a facilitação no local indicado pela pista emerge lentamente, atinge seu ápice após $300 \mathrm{~ms}$ de AIE (CHEAL, LYON e HUBBARD, 1991) e permanece constante por várias centenas de milissegundos após esta AIE (CHEAL, 1993; CHEAL e LYON, 1991; CHEAL, LYON e HUBBARD, 1991; LYON, 1990) enquanto a inibição no local oposto àquele indicado pista emerge progressivamente após 100 ms de AIE (WARNER, JUOLA e KOSHINO, 1990).

Estudos com potenciais relacionados ao evento têm sido muito utilizados para caracterizar o curso temporal do processamento visual em humanos e sua modulação pela atenção espacial. O potencial relacionado ao evento consiste de várias deflexões de voltagem, que começam $50 \mathrm{~ms}$ após o início de um estímulo visual, chamadas de C1 (50-90 ms), P1 (80-130 ms) e N1 (140-200 ms). A orientação prévia da atenção para o local deste estímulo, tipicamente resulta em uma melhora na amplitude dos componentes $\mathrm{P} 1$ e N1 evocados por ele. Isto sugere que a atenção espacial exerce um ganho ou amplificação seletiva no fluxo de informação sensorial em vias visuais extraestriadas, iniciando aos $80 \mathrm{~ms}$ depois do início do estímulo. Para a atenção a características não espaciais como cor, movimento ou forma, diferenças no padrão dos potenciais relacionados ao evento são evidenciadas com latências de 100-150 ms (HILLYARD e ANLLO-VENTO, 1998).

Vários autores têm proposto possíveis mecanismos neurofisiológicos para explicar os mecanismos atencionais. Lamme e Roelfsema (2000) diferenciam os processos pré-atencionais dos atencionais utilizando o modelo de processamento visual anterógrado e recorrente. Uma análise da latência de resposta mostra que quando um estímulo visual é apresentado ao indivíduo, os sinais neuronais são rapidamente encaminhados para um grande número de áreas visuais ("onda" anterógrada). Contudo, a atividade dos neurônios corticais não é determinada unicamente por esta "onda" anterógrada. Conexões recorrentes (horizontais entre estas áreas visuais e retrógradas das áreas hierarquicamente superiores para as inferiores) podem resultar em mudanças dinâmicas na sintonia de um neurônio. É sugerido que a "onda" anterógrada da informação estaria relacionada com os 
processos pré-atencionais, enquanto que uma parte das "ondas" recorrentes estaria relacionada com os processos atencionais.

Vidyasagar (1999) propõe um modelo neural de holofote atencional. Neste modelo, quando a informação atinge o córtex visual primário é canalizada para duas vias: uma via dorsal (com direção ao córtex parietal) que veicula a maioria dos sinais da via magnocelular e uma via ventral (com direção ao córtex temporal) que veicula a maioria dos sinais da via parvocelular. O holofote atencional seria originado na via dorsal e então direcionado para a via ventral, com a função de facilitar uma busca visual e construção da imagem. Esta influência da via dorsal sobre a via ventral é possível, pelo fato do canal magnocelular transiente ser mais rápido do que o canal parvocelular sustentado. O nível que o foco é direcionado depende do tamanho e quantidade dos estímulos alvos no campo visual. Se existe um pequeno número de estímulos alvos desordenadamente distribuídos na cena visual, o foco facilitador operaria nas regiões mais precoces como V1. Se os estímulos alvos são grandes e poucos, o foco seria direcionado às regiões mais altas como V2, V4, área têmporooccipital ou área temporal.

Vários estudos de imageamento cerebral têm tentado determinar quais são as áreas envolvidas na atenção automática e na atenção voluntária. Alguns estudos referem ampla sobreposição destas áreas (CORBETTA et al., 1993; KIM et al. 1999; NOBRE et al., 1997; PEELEN, HESLENFELD e THEEUWES, 2004) enquanto outros estudos referem áreas distintas de ativação (CORBETTA e SHULMAN, 2002; MAYER et al., 2004). Kim et al. (1999) propõem que as duas formas de direcionamento da atenção utilizam uma rede comum de regiões corticais e subcorticais. Eles evidenciaram atividade cerebral, tanto para a atenção automática quanto para a atenção voluntária, no campo ocular frontal, córtex parietal posterior, giro cingulado, putamem e tálamo. Adicionalmente, ativações ocorreram na insula anterior, córtex prefrontal dorsolateral, córtex pré-frontal, córtex têmporo-occipital, área motora suplementar e cerebelo. Porém, foram observadas ativações mais pronunciadas na tarefa de direcionamento da atenção voluntária do que na tarefa de direcionamento da atenção automática.

Corbetta e Shulman (2002) propõem que as redes neurais ativadas na atenção voluntária são parcialmente distintas daquelas ativadas na atenção automática. Os autores evidenciaram um sistema dorsal, envolvendo a região dorsal do córtex parietal posterior e o córtex pré-frontal e um sistema ventral, centrado nos 
córtices temporo-parietal e frontal anterior. O sistema dorsal seria responsável pela seleção cognitiva da informação sensorial e respectivas respostas na atenção voluntária. O sistema ventral seria recrutado durante a detecção de eventos relevantes, ou salientes, do ambiente na atenção automática.

Há evidências de que os mecanismos responsáveis pela atenção automática e voluntária estariam assimetricamente representados nos dois hemisférios cerebrais. Um clássico estudo de Corbetta et al., (1993) bastante citado, com tomografia por emissão de pósitron em tarefas que envolviam tanto a atenção automática quanto a atenção voluntária, demonstrou que o lobo parietal superior direito apresentava um aumento do fluxo sangüíneo quando a atenção era direcionada tanto para o lado esquerdo quanto para o lado direito do espaço, enquanto que o lobo parietal esquerdo apresentava um aumento do fluxo sangüíneo apenas quando a atenção era direcionada para o lado direito do espaço. Mesulam e colaboradores (KIM et al., 1999), usando ressonância magnética funcional, encontraram uma ativação no córtex parietal posterior mais extensa no hemisfério direito (principalmente no sulco intraparietal direito) durante o direcionamento tanto da atenção automática quanto da voluntária. Porém, a assimetria foi maior na tarefa que envolvia a atenção automática do que na tarefa que envolvia a atenção voluntária. Esta ativação mais extensa do hemisfério direito é consistente com a noção de seu maior envolvimento na distribuição da atenção.

Em um estudo mais recente de Corbetta, Kincade e Shulman (2002), com ressonância magnética funcional, envolvendo somente atenção voluntária, evidenciou que sistema dorsal, formado pelo sulco intraparietal ventral (vIP) e o campo ocular frontal (FEF), foi fortemente ativado de acordo com direção da pista. Pistas para a esquerda, que produziam uma mudança da atenção para o campo visual esquerdo, evocavam respostas mais fortes no hemisfério direito. Pistas para a direita, que produziram uma mudança da atenção para o campo visual direito, evocavam respostas em ambos os hemisférios. Esta modulação direcional, específica para o período em que a pista era apresentada, foi relacionada ao controle da alocação e manutenção da atenção voluntária. Porém, uma rede separada no hemisfério direito, o sistema dorsal, formado principalmente pela junção temporo-parietal direita (JTP), foi ativado quando o alvo aparecia no local oposto ao indicado pela pista. Esta ativação, específica para o período em que o alvo era apresentado, mostrou-se independente do hemicampo visual em que ele aparecia. 
Foi proposto que o sistema ventral (JTP) seria importante para a reorientação da atenção a eventos inesperados e que os dois sistemas, o dorsal (IP-FEF) e o ventral (JTP) se interagiriam. Uma possibilidade é que o sistema ventral serviria como um sistema de alerta que detecta estímulos comportamentalmente relevantes no ambiente, mas que por não ser equipado com sensores de alta resolução espacial, quando um estímulo relevante inesperado fosse detectado, a localização precisa deste estímulo dependeria do sistema dorsal. O sistema dorsal atuaria como um circuito interruptor da atividade cognitiva em funcionamento quando um estímulo comportamentalmente relevante fosse detectado. Isto é, quando se detecta um estímulo inesperado, torna-se necessário interromper a ação corrente do mecanismo atencional para redireciná-lo ao novo estímulo (CORBETTA e SHULMAN, 2002).

Um estudo em que foi utilizada a estimulação magnética transcraniana confirma a existência de uma assimetria inter-hemisférica favorecendo o controle atencional do hemisfério direito para os dois lados do espaço (MURI et al. 2002). Neste estudo, envolvendo a atenção voluntária, dois símbolos eram apresentados simultaneamente, um no lado direito do espaço e o outro no lado esquerdo. A tarefa consistia em identificar o padrão do estímulo apresentado em quatro possíveis combinações. A estimulação magnética foi aplicada durante 100 ms no córtex parietal posterior direito ou no córtex parietal posterior esquerdo, em diferentes intervalos de tempo (120, 270 e 520 ms), depois do início dos estímulos. A estimulação no córtex parietal posterior direito, após o intervalo de $270 \mathrm{~ms}$, levou a um aumento significativo na porcentagem de erros no lado contralateral à estimulação. Nenhum aumento significativo no número de erros foi encontrado com a estimulação magnética no córtex parietal esquerdo.

Além dos estudos referidos acima, trabalhos realizados em pacientes que sofreram lesões centrais também sugerem uma assimetria inter-hemisférica dos mecanismos atencionais. A Síndrome da Heminegligência tende a causar distúrbios atencionais mais severos e mais freqüentes após lesão no hemisfério direito do que no hemisfério esquerdo. Assim, muitos pacientes com lesão hemisférica direita passam a ignorar estímulos visuais, auditivos e táteis no lado contralateral (MESULAM, 1999; BRADSHAW e NETTLETON, 1983; KERKHOFF, 2001; NATALE, 2005). Estes indivíduos são extremamente lentos para responder a estímulos alvos visuais localizados no lado contralateral à lesão (POSNER et al., 1984; PETERSEN, ROBINSON e CURRIE, 1989; POSNER e DRIVER, 1992) devido aparentemente a um prejuízo tanto para a atenção automática quanto para a atenção voluntária 
(FRIDRICH, RAFAL e BECK, 1998). Foi demonstrado, em registros eletrofisiológicos, que pacientes com heminegligência, após lesão no hemisfério direito, apresentam potenciais relacionados ao evento, com maiores latências e mais baixas amplitudes para estímulos visuais que aparecem no lado contralateral à lesão (ANGELELLI, LUCA e SPINELLI, 1996). Segundo Mesulam (1999), o hemisfério direito contém uma rede neural para direcionar a atenção para os dois lados do espaço enquanto que o hemisfério esquerdo contém uma rede para direcionar a atenção apenas para o lado direito. Estas redes neuronais incluiriam o córtex prémotor lateral, o córtex parietal posterior, o córtex cingulado anterior e subcorticalmente, os gânglios da base e o tálamo. As lesões no hemisfério direito freqüentemente produziriam heminegligência à esquerda, enquanto que lesões no hemisfério esquerdo raramente produziriam heminegligência à direita devido à maior capacidade do sistema atencional do hemisfério direito, que com sua orientação bilateral, compensaria os prejuízos causados pela lesão do hemisfério esquerdo.

De uma forma geral a maioria dos estudos baseados na análise da atividade cerebral normal e efeitos da estimulação magnética transcraniana e de lesões centrais sugere uma assimetria inter-hemisférica dos mecanismos atencionais. Considerando que ambos os hemisférios controlam a atenção para o lado direito do espaço e somente o hemisfério direito controla a atenção para o lado esquerdo do espaço, a existência de um favorecimento do lado direito do espaço torna-se possível de imaginar.

Existem algumas evidências que a assimetria dos mecanismos envolvidos na atenção automática e na atenção voluntária possa ser expressa no comportamento. A maioria dos estudos que analizaram a atenção automática demonstraram uma assimetria da atenção automática favorecendo o lado direito do espaço. Steinman, Steinman e Lehmkuhle (1995) avaliaram a ilusão de que uma linha contínua apresentada imediatamente após um estímulo precedente abrupto. Eles observaram que a linha cresce em direção oposta a este estímulo quando localizada próxima a ele e na direção deste estímulo quando localizada distante dele. O primeiro efeito resultaria da facilitação do processamento sensorial pela atenção no local do estímulo abrupto. O segundo efeito resultaria da inibição do processamento sensorial pela atenção nos locais distantes dele. O efeito facilitador foi maior quando o estímulo precedente aparecia no lado esquerdo do espaço. O efeito 
inibidor foi, no entanto, maior quando o estímulo abrupto aparecia no lado direito do espaço. Pollman $(1996,2000)$ apresentou simultaneamente um alvo visual em um lado do espaço e um distraidor saliente no outro lado do espaço. Esperava-se que a atenção fosse inicialmente direcionada para o distraidor e depois para o alvo, aumentando o tempo de reação. Foi observado que os tempos de reação ao alvo foram maiores quando o distraidor saliente aparecia no lado direito e o alvo no lado esquerdo do que o inverso. Evert e Oscar-Berman (2001) usaram estímulos precedentes periféricos para direcionar a atenção para o lado direito ou para o lado esquerdo do espaço. $O$ alvo foi apresentado simultaneamente a um distraidor contralateral, $165 \mathrm{~ms}$ depois do início do estímulo precedente. Os autores não observaram diferenças interlaterais na discriminabilidade do alvo quando ele aparecia no mesmo local do estímulo precedente; no local oposto ao do estímulo precedente, a discriminabilidade do alvo no lado direito foi maior do que no lado esquerdo. Investigamos recentemente (CASTRO-BARROS, 2004) se a existência de uma assimetria no efeito atencional automático poderia variar conforme o tipo de tarefa utilizada. Foram utilizadas dois diferentes tipos de tarefas, uma tarefas de tempo de reação simples, que envolvia apenas a discriminação do local do alvo e uma tarefa de tempo de reação vai/não-vai, que envolvia além da discriminação do local também da forma do alvo. Um estímulo precedente periférico não informativo foi utilizado para direcionar a atenção. $O$ tempo de reação para o alvo no mesmo local ou no local horizontalmente oposto foi avaliado. As AIE foram 34, 50, 67, 83 e $100 \mathrm{~ms}$. Um importante efeito do estímulo precedente foi observado bem precocemente (aos $50 \mathrm{~ms}$ ) para ambas as tarefas. Este efeito foi maior quando o estímulo precedente aparecia no lado direito do que quando aparecia no lado esquerdo do espaço para a AIE de $100 \mathrm{~ms}$. Nossos resultados indicam que a atenção automática tende a favorecer a lado direito do espaço particularmente quando a discriminação da forma do alvo é requerida.

Já os estudos que analizaram a atenção voluntária demonstraram resultados bastante contraditórios. Evert et al., (2003) estudaram a atenção voluntária por meio de pistas centrais (válida, inválida e neutra) em tarefas com diferentes demandas perceptuais. Os voluntários, depois de direcionar a atenção, deveriam responder a um alvo que poderia aparecer no lado direito ou no lado esquerdo do espaço. Eles encontraram que o custo (diferença entre o tempo de reação ao alvo quando a pista era inválida e o tempo de reação quando a pista era neutra) foi mínimo quando o alvo aparecia no lado esquerdo em relação a quando aparecia no lado direito; não 
havia diferença interlateral para o benefício atencional (diferença entre o tempo de reação ao alvo quando a pista era neutra e o tempo de reação quando a pista era válida). Esta assimetria estava presente apenas nas tarefas com alta demanda perceptual e provavelmente foi devido a um favorecimento do lado esquerdo pelos mecanismos atencionais.

Diferentemente, uma assimetria favorecendo o lado direito do espaço foi encontrada por Rhodes e Robertson (2002). Eles também utilizaram estímulos pistas centrais para direcionar a atenção e encontraram que quando a pista era válida, as respostas eram mais rápidas para alvos no lado direito do que para alvos no lado esquerdo; não havendo diferença interlateral quando a pista era inválida (Experimento 1).

Barthelémy e Boulinguez (2002) utilizando procedimento semelhante encontraram que quando a pista era inválida e neutra, o tempo de reação foi menor quando a resposta ao alvo era dada com a mão esquerda do que com a mão direita, Quando a pista era válida não houve diferença significativa entre as mãos. Eles sugerem um favorecimento da mão esquerda pelos mecanismos atencionais, sendo esta vantagem, devido a uma maior dominância do hemisfério direito para o direcionamento da atenção visuoespacial. Os resultados destes três grupos de autores (RHODES e ROBERTSON, 2002; EVERT et al., 2003; BARTHELÉMY e BOULINGUEZ, 2002) além de contraditórios, são difíceis de interpretar, uma vez que eles consideraram para efeito de comparação, o lado da apresentação do alvo ao invés do lado de apresentação da pista. Também foi utilizada uma medida de benefício e custo para avaliar as diferenças interlaterais (EVERT et al., 2003); vários autores criticam a real neutralidade desta condição neutra (JONIDES e MACK, 1984 e HAWKINS et al., 1990). Além disto, Evert et al., (2003), RHODES e ROBERTSON (2002) usaram estímulos lingüísticos o que pode ter introduzido uma outra importante fonte de assimetria interlateral.

O objetivo deste estudo foi determinar a exata direção da assimetria interlateral da atenção visuoespacial voluntária. Para tanto seu curso temporal foi avaliado com um procedimento experimental bem padronizado na literatura para quantificar a atenção voluntária, chamado de Procedimento de Posner (veja a cima, POSNER e COHEN, 1984).

Três experimentos, subdivididos em duas partes, foram realizados. O curso temporal do efeito atencional foi evoluído em uma tarefa de escolha simples, Experimentos 1A, 2A e 3A, e em uma tarefa de escolha vai/não-vai, Experimentos 
1B, 2B e 3B. Nos Experimentos 1A e 1B, as AIE foram 100, 200, 300, 400 e $500 \mathrm{~ms}$ e as pistas podiam ser válidas ou inválidas. Nos Experimentos $2 \mathrm{~A}$ e $2 \mathrm{~B}$, as AIE foram 100, 300 e 500 e as pistas podiam ser válidas, inválidas ou bilaterais. Os Experimentos $3 \mathrm{~A}$ e $3 \mathrm{~B}$ foram semelhantes, respectivamente, aos Experimentos $2 \mathrm{~A} e$ $2 \mathrm{~B}$, porém adicionalmente ao alvo foi apresentado um distraidor contralateralmente. A utilização da tarefa de escolha simples e da tarefa de escolha vai/não-vai baseouse na idéia de que a assimetria poderia variar entre elas. Talvez esta assimetria fosse menor na tarefa de escolha simples que envolveria principalmente mecanismos de discriminação do local do alvo, do que a tarefa de escolha vai/nãovai que envolveria além dos mecanismos de discriminação do local, mecanismos de discriminação da forma do alvo.

Uma assimetria interlateral modesta do efeito atencional favorecendo o lado direito do espaço foi observada, para a AIE mais longa (500 ms), no experimento que requeria uma discriminação mais fácil da forma do alvo (Experimento $2 \mathrm{~B}$ ). Também foi observada uma assimetria interlateral importante favorecendo o mesmo lado, para todas as $\operatorname{AIE}(100,300$ e $500 \mathrm{~ms})$, nos dois experimentos que requeriam uma discriminação mais difícil da forma do alvo (Experimentos $3 \mathrm{~A}$ e 3B). A observação de que há uma assimetria interlateral no curso temporal da atenção voluntária, particularmente quando a necessidade de discriminação da forma do alvo é requerida, é original. De grande interesse, ela pode ser explicada por um Modelo de Assimetria Interlateral que leva em conta os modelos neurofisiológicos da atenção (VIDYASAGAR, 1999 e LAMME E ROELFSEMA, 2000) e o modelo de assimetria inter-hemisféricas dos mecanismos atencionais (MESULAM, 1999). 


\section{Conclusão}

Nós demonstramos o aparecimento de um acentuado efeito atencional já aos $100 \mathrm{~ms}$ de AIE. Este efeito praticamente não se alterou nas outras assincronias, em ambas as tarefas de escolha simples e escolha vai/não-vai.

Uma assimetria interlateral do efeito atencional favorecendo o lado direito do espaço foi observada, para a assincronia mais longa (500 ms), em um experimento que requeria uma discriminação relativamente mais fácil da forma do alvo (Experimento 2B). Também foi observada uma assimetria interlateral favorecendo o mesmo lado, porém em todas as assincronias (100, 300 e $500 \mathrm{~ms})$, em dois experimentos que requeriam uma discriminação relativamente mais difícil da forma do alvo (Experimentos $3 \mathrm{~A}$ e $3 \mathrm{~B}$ ). No primeiro caso a assimetria foi modesta, nos outros dois, ela foi acentuada.

Acreditamos que esta assimetria interlateral, favorecendo o lado direito do espaço, seja decorrente das assimetrias inter-hemisféricas dos mecanismos atencionais. 


\section{REFERÊNCIAS BIBLIOGRÁFICAS}

ANGELELLI, P.; LUCA, M.; SPINELLE, D. Early visual processing in neglect patients: A study with steady-state VEPs. Neuropychologia, v. 34, p. 1151-1157, 1996.

AWH, E.; PASHLER, H. Evidence for split attention foci. Journal Experimental Psychology Human Perception Performance, v. 26, p. 834-846, 2000.

BAND, G.P.H.; RIDDERINKHOF, K.R; VAN DER MOLEN, M.W. Speed-accuracy modulation in case of conflict: the roles of activation and inhibition. Psychological Research, v. 67, p. 266-279, 2003.

BARTHELÉMY, S.; BOULINGUES, P. Orienting visuospatial attention generates manual reaction time asymmetries in target detection and pointing. Behavioral Brain Research, v. 133, p. 109-116, 2002.

BENSO, F.; TURATTO, M.; MASCETTI, G.G.; UMILTÀ C. The time course of attentional focusing. European Journal of Cognitive Psychology, v.10, p. 373388, 1998.

BERGER, A.; HENIK, A.; RAFAL, R. Competition between endogenous and exogenous orienting of visual attention. Journal of Experimental Psychology: general, v. 134, p. 207-221, 2005.

BRADSHAW, J.L.; NETTLETON, N.C. Human Cerebral Asymmetry. New Jersey: Prentice-Hall, 1983.

CASTIELLO, U.; UMILTÀ, C. Size of the attentional focus ad efficiency of processing. Acta Psychologica, v. 73, p. 195-209, 1990.

Psychology, v. 18, p. 837-848, 1992.

Splitting focal attention. Journal of Experimental

CASTRO-BARROS, B.A. Há assimetria interlateral na capacidade de orientação da atenção visuespacial automática? 125 f. Dissertação (Mestrado em Ciências) - Instituto de Ciências Biomédicas, Universidade de São Paulo, São Paulo, 2004.

CHEAL, M.; LYON, D. R. Central and Peripheral precuing of forced-choice discrimination. The Quarterly Journal of Experimental Psychology, v. 43 A, p. 859-880, 1991.

Benefits from attention depend on the target type in location-precued discrimination. Acta Psychologica, v. 81, p. 243-267, 1992.

CHEAL, M.; LYON D. R.; HUBBARD D. C. Does attention have different effects on line orientation and line arrangement? The Quarterly Journal of Experimental Psychology, v. 43 A, p. 825-857, 1991. 
CHEAL, M. Rapid performance improvement across short precue-target delays is most likely due to effects of attention and not masking from peripheral precues. Acta Psychologica, v. 83, p. 79-86, 1993.

CORBETTA, M.; MIEZIN, F.M.; SHULMAN, G.L. A PET study of visuospatial attention. The Journal of Neuroscience, v. 13, p. 1202-1226, 1993.

CORBETTA, M.; KINCADE, M.; SHULMAN, G.L. Neural systems for visual orienting and their relationships to spatial working memory. Journal of Cognitive Neuroscience, v. 14. p. 508-523, 2002.

CORBETTA, M.; SHULMAN, L. Control of goal-directed and stimulus-friven attention in the brain. Nature Reviews, v. 3, p. 201-215, 2002.

COULL, J. T.; NOBRE, A. C. Where and when to pay attention: the neural systems for directing attention to spatial locations and to time intervals as revealed by both PET and fMRI. The Journal of Neuroscience, v. 18, p. 74267435, 1998.

DAVIDSON, H.; CAVE, K.; SELLNER, D. Differences in visual attention and task interference between males and females reflect differences in brain laterality. Neuropysichologia, v. 38, p. 508-519, 2000.

DOALLO, $\mathrm{S}$ et al. The time course of the effects of central and peripheral cues on visual processing: an event-related potential study. Clinical Neurophysiology, $v$. 115, p. 199-210, 2004.

Modulation of the visual N1 component of event related potential by central and peripheral cueing. Clinical Neurophysiology, v. 116, 807-820, 2005.

DOSHER, B.A.; LU, Z.L Noise exclusion in spatial attention. Psychological Science, v. 11, p. 139-146, 2000.

EVERT, D.L.; OSCAR-BERMAN, M.O. Selective attentional processing and the right hemisphere: effects of aging and alcoholism. Neuropsychology, v. 15, p. 452-461, 2001.

EVERT, D.L. et al. Hemispheric asymmetries for selective attention apparent only with increased task demands in health participants. Brain and Cognition, v. 53, p. 34-41, 2003.

FRIEDRICH, F.J.; RAFAL, R.D.; BECK, D. Spatial Attention deficts in human: a comparison of superior parietal and temporal-parietal junction lesions. Neuropychology, v. 12, p. 193-207, 1998.

GAZZANIGA, M. S.; IVRY, R.B.; MANGUN, G. Neurociência Cognitiva: a biologia de mente. Porto Alegre: ArtMed, 2006.

GOODALE, M.A.; MILNER, A.D. Separate visual pathways for perception and action. Trends in Neuroscience, v. 15, p. 20-25, 1992. 
HAUSMANN, M. Hemispheric asymmetry in spatial attention across the menstrual cycle. Neuropysichologia, v. 43, p. 1559-1567, 2005.

HAWKINS, H.L.; HILLYARD, S. A.; LUCK, S.J.; MOULOUA, M.; DOWNING, C.J.; WOODWARD D.P. Visual attention modulates signal detectability. Journal Experimental Psychology Human Percept Performance, v. 16, p. 802-811, 1990.

HYLLYARD, S.; ANLLO-VENTO, S. Event-related brain potentials in the study of visual selective attention. Proceedings of the National Academy of Sciences of the United States of America, v. 95, p. 781-787, 1998.

IACCINO, J.F. Left Brain-Right Brain Differences: Inquiries, Evidence, and New Approaches. New Jersey: Lawrence Erlbaum Associates, 1993.

YAMAGUCHI, S.; YAMAGATA, S.; KOBAYASHI, S. Cerebral asymmetry of the "Top-Down" Allocation of attention to global and local features. The journal of Neuroscience, v. 20, p. 1-5, 2000.

JONIDES, J. Voluntary versus automatic control over the mind's eye's movement. In: Long, J. and Baddeley, A., Attention and performance. New Jersey: Lawrence Erlbaum, 1981. p. 187-203.

JONIDES, J.; MACK, R. On the cost and benefit of cost and benefit. Psychological Bulletin, v. 96, p. 29-44, 1884.

KRAFT, A. et al. Interactions beween task difficulty and hemispheric distribution of attended location: implications for the spitting attention debate. Cognitive Brain Reseach, v. 24, p. 19-32, 2005.

KERHOFF, G. Spatial hemineglect in humans. Progress in Neurobiology, v. 63, p.1-27, 2001.

KITTERLE, F.L.; CHRISTMAN, S; HELLIGE, J.B. Hemispheric differences are found in the identification, but not the detection, of low versus high spatial frequencies. Perception and Psychophysics, v. 48, p. 297-306, 1990.

KIM, Y.H.; GITELMAN, D.R.; NOBRE, A.C. PARRISH, T.B.; LABAR, K.S.; MESULAM, M.M. The large-scale neural network for spatial attention displays multifunctional overlap but differential asymmetry. Neurolmage, v. 9; p. 269-277, 1999.

LAMME, V. A. F.; ROELFSEMA, P. R. The Distinct modes of vision offered by feedforward and recurrent processing. Trends Neuroscience, v. 23, p. 571-579, 2000.

LIEDERMAN, J. Determinants of the enhancement of the right visual field advantage by bilateral vs unilateral stimuli. Cortex, v. 22, p. 553-565, 1986.

LYON, D. R. Large and rapid improvement in form discrimination accuracy following a location precue. Acta Psychologica, v. 73, p. 69-82, 1990. 
MALINOWSKI, P. et al. The influence of response competition on cerebral asymmetries for processing hierarchical stimuli reveled by ERP recordings. Experimental Brain Research, v. 144, p. 36-139, 2002.

MATTINGLEY, J.B.; DRIVER, J.; BESCHIN, N.; ROBERTSON, I.H. Attention competition between modalities: extinction between touch and vision after right hemisphere damage. Neuropsychologia, v. 35, p. 867-880, 1987.

MAYER, A.R. et al. Neural networks underlying endogenous and exogenous visual-spatial orienting. Neuroimage, v. 23, p. 534-541, 2004.

MARZI, C.A.; BISIACCHI, P.; NICOLETTI, R. Is interhemispheric transfer of visuomotor information asymmetric? Evidence from a meta-analysis. Neuropsychologia, v. 29, p. 1163-1177, 1991.

MESULAM, M. M. Spatial attention and neglect: parietal, frontal and cingulated contributions to the mental representation and attentional targeting of salient extrapersonal events. Philosophical Transactions of Royal Society London, v. 354, p. 1325-1346, 1999.

MÜRI, R.M. et al. Hemispheric asymmetry in visuoespatial attention assessed with transcranial magnetic stimulation. Experimental Brain Research, v. 143, p. 426-430, 2002.

MÜLLER, M.M. et al. Sustained division of the attentional spotlight. Letters to Nature, v. 429, p. 309-312, 2003.

NAKAYAMA, K.; MACKEBEN, M. Sustained and transient components of focal visual attention. Vision Research, v. 29, p. 1631-1647, 1989.

NATALE, E. et al. What kind of visual spatial attention is impaired in neglect. Neuropsychologia, v. 43, p. 1072-1085, 2005.

NOBRE, A.C. et al. Functional localization of the system for visuoespatial attention using positron emission tomography. Brain, v. 120, p. 5515-533, 1997.

OLDFIELD, R.C. The assessment and analysis of handedness: The Einburgh Inventory. Neuropsychologia, v. 9, p. 97-113, 1971.

PEELEN, M.V; HESLENFELD, D J.; THEEUWES, J. Endogenous and exogenous attention shifts are mediated by the same large-scale neural network. Neuroimage, v. 22, p. 822-830, 2004.

PETERSEN, S.E.; ROBINSON, D.L.; CURRIE, J.N. Influences of lesions of parietal cortex on visual spatial attention en humans. Experimental Brain Research, v. 76, p. 276-280, 1989.

POLLMANN, S. A pop-out induced extinction-like phenomenon in neurologically intact subjects. Neuropsychologia, v. 34, p. 413-425, 1996.

Extinction-like effects in normals: independence of localization and response selection. Brain and Cognition, v. 44 p. 324-341, 2000. 
POSNER, M.I. Chronometric exploration of mind. New Jersey: Hillsdale Erlbaum, 1978.

POSNER, M. I.; COHEN, Y. Components of visual orienting. In: BOUMA, H.; BOUWHUIS, G.G. (Ed.). Attention and performance. New Jersey: Hillsdale Erlbaum, 1984. p. 531-556.

POSNER, M.I.; RAICHLE, M.E. Networks of Attention. In: Imagens of Mind. New York: Scientific American Library, 1994. p. 154-179.

POSNER, M.I. et al. Effects of parietal injury on covert orienting of attention. The Journal of Neuroscience. v. 4, p. 1863-1874, 1984.

POSNER, M.I.; DRIVER, J. The neurobiology of selective attention. Current Biology, v. 2, p. 165-169, 1992.

POSNER, M.I.; SNYDER, C.R.R.; DAVIDSON, B.J. Attention and detection of signals. Journal of Experimental Psychology: general, v. 109, p.160-174, 1980.

RHODES, D.; ROBERTSON, L. Visual field asymmetries and allocation of attention in visual scenes. Brain and Cognition, v. 50, p. 95-115, 2002.

STEINMAN, S. B.; STEINMAN, B. A. Vision and attention: current models of visual attention. Optometry and Vision Science, v. 75, p. 146-155, 1998.

STEINMAN, S.B.; STEINMAN, B.A.; LEHMKUHLE, S. Visual attention mechanisms show a center-surround organization. Vision Research, v. 35, p. 1859-1869, 1995.

TURATTO, M. et al. Automatic and voluntary focusing of attention. Perception and Psychophysics, v. 62, p. 935-952, 2000.

VIDYASAGAR, T. R. A Neuronal model of attentional spotlight: parietal guiding the temporal. Brain Research Reviews, v. 30, p. 6-76, 1999.

WARNER, C.B.; JUOLA, J.F.; KOSHINO, H. Voluntary allocation versus automatic capture of visual attention. Perception and Psychophysics, v. 48, p. 243-251, 1990.

WRIGHT, R.D.; RICHARD, C.M.; MCDONALD J.J. Neutral location cues and cost/benefit analysis of visual attention shifts. Canadian Journal of Experimental Psychology, v. 49, p. 540-548, 1995. 
